\title{
AutoDiagn: An Automated Real-time Diagnosis Framework for Big Data Systems
}

\author{
Umit Demirbaga, Zhenyu Wen* Member, IEEE, Ayman Noor, Karan Mitra, Member, IEEE, Khaled \\ Alwasel, Saurabh Garg, Albert Zomaya, Fellow, IEEE, Rajiv Ranjan, Senior Member, IEEE
}

\begin{abstract}
Big data processing systems, such as Hadoop and Spark, usually work in large-scale, highly-concurrent, and multi-tenant environments that can easily cause hardware and software malfunctions or failures, thereby leading to performance degradation. Several systems and methods exist to detect big data processing systems' performance degradation, perform root-cause analysis, and even overcome the issues causing such degradation. However, these solutions focus on specific problems such as stragglers and inefficient resource utilization. There is a lack of a generic and extensible framework to support the real-time diagnosis of big data systems. In this paper, we propose, develop and validate AutoDiagn. This generic and flexible framework provides holistic monitoring of a big data system while detecting performance degradation and enabling root-cause analysis. We present an implementation and evaluation of AutoDiagn that interacts with a Hadoop cluster deployed on a public cloud and tested with real-world benchmark applications. Experimental results show that AutoDiagn can offer a high accuracy root-cause analysis framework, at the same time as offering a small resource footprint, high throughput and low latency.
\end{abstract}

Index Terms-Online analysis, Outliers, Root-cause analysis, Big data systems, QoS, Hadoop, Performance

\section{INTRODUCTION}

The rapid surge of data generated through sectors like social media, financial services and industries has led to the emergence of big data systems. Big data systems enable the processing of massive amounts of data in relatively short time frames. For instance, the Netflix big data pipeline processes approximately 500 billion events and 1.3 petabytes (PB) of data per day, further, during peak hours, it processes approximately 11 million events and 24 gigabytes (GB) of data on a per-second basis. Facebook has one of the largest data warehouses in the world, capable of executing more than 30,000 queries over 300 PB data every day. However, the enormousness and complexity of the big data system runs in heterogeneous computing resources, multiple tenant environments, as well as has many concurrent execution of big data processing tasks, which makes it a challenge to utilize the big data systems efficiently and reliably[1]. For example, Fig. 1 shows that the performance degrades at least $10 \%$ when the resources are not utilized efficiently with Setting 2 .

- U. Demirbaga is with Newcastle University, United Kingdom and Bartin University, Turkey. E-mail: u.demirbaga2@newcastle.ac.uk

- Z. Wen is with Newcastle University, United Kingdom. E-mail: zhenyu.wen@newcastle.ac.uk, corresponding author.

- A. Noor is with Newcastle University, United Kingdom and Taibah University, Saudi Arabia.E-mail: anoor@taibahu.edu.sa

- K. Mitra is with Lulea University of Technology, Sweden. E-mail: karan.mitra@ltu.se

- K. Alwasel is with Newcastle University, United Kingdom and Saudi Electronic University, Saudi Arabia. E-mail: kalwasel@gmail.com

- S. Garg is with University of Tasmania, Australia. Email:Saurabh.Garg@utas.edu.au

- A. Zomaya is with Sydney University, Australia, E-mail: albert.zomaya@sydney.edu.au

- R. Ranjan is with Newcastle University, United Kingdom. E-mail: raj.ranjan@newcastle.ac.uk

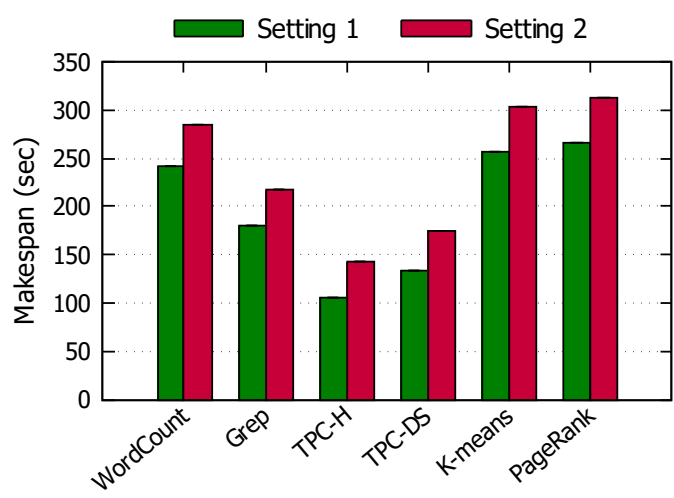

Big data applications

Fig. 1. Six big data applications are executed in a cloud-based Hadoop cluster with two settings: 1) the input data and jobs are allocated in the same node; 2) the input data and jobs are allocated in different nodes. In Setting 2, the execution time of each application is delayed by transmitting data across nodes.

To overcome this, it is imperative to continuously monitor and analyze all available system resources at all times in a systematic, holistic and automated manner. These resources include CPU, memory, network, I/O and the big data processing software components.

Most of the commercial [2][3]|4] and academic big data monitoring systems mainly focus on visualizing task progress, and the system's resource utilization [5]. However, they do not focus on the interaction between multiple factors and performing root-cause analysis for performance degradation [6]|7]. Moreover, works such as [8], [9] aim to find the best parameters to optimize the performance of

Manuscript received ???; revised ??? 
big data processing systems, they do not focus on the rootcause analysis that may indicate the viable reasons behind performance degradation and may provide intuitions for parameter tweaking.

Mantri [10] presents a systematic method that categorizes the main reasons causing outliers in a big data system. The authors' work was focused on the MapReduce programming framework in the Hadoop system; they do not discuss how Mantri can be applied to other big processing frameworks (e.g., Apache Spark ${ }^{1}$, and Apache Flink ${ }^{2}$ ). Garraghan et al. [11] proposed an online solution to detect longtail issues in a distributed system. However, these solutions were built for specific scenarios with much scope left for analyzing a variety of problems that can exist in a large scale big data processing system.

To the best of our knowledge, there is a lack of a generic and comprehensive solution for the detection of a wide range of anomalies and performance of root-cause analysis in big data systems. Developing a general and extensible framework for diagnosing a big data system is not trivial. It requires well-defined requirements which could enable the broader adoption of root-cause analysis for the big data systems, flexible APIs to interact with an underlying monitoring system and integration of multiple solutions for detecting performance reduction problems while enabling the automatic root-cause analysis. In this paper, we tackle this research gap, and design and develop AutoDiagn to automatically detect performance degradation and inefficient resource utilization problems, while providing an online detection and semi-online root-cause analysis for a big data system. Further, it is designed as a microservice architecture that offers the flexibility to plug a new detection and root-cause analysis module for various types of big data systems.

The contributions of this paper are as follows:

- An online and generic framework: We develop a general framework called AutoDiagn which can be adapted for the detection of a wide range of performance degradation problems while pinpointing their rootcauses in big data systems.

- A case study: We develop a novel real-time stream processing method to detect symptoms regarding outliers in a big data system. After that, we develop a set of query APIs to analyze the reasons that cause the outlier regarding a task.

- A comprehensive evaluation: We evaluate the feasibility, scalability and accuracy of AutoDiagn through a set of real-world benchmarks over a real-world cloud cluster.

The paper is organized as follows. The design requirements and idea are outlined in \$2 In \$3. we illustrate the high-level system architecture. $\$ 4$ presents a case study that we implemented and the case study is evaluated in \$5 \$6 discusses the limitations of this paper and highlights our further work. Before drawing a conclusion in $\$ 8$, we discuss the related work in $\$ 7$

1.https://spark.apache.org/

2. https://flink.apache.org/

\section{REQUiREMENTS AND DESIGN IDEA}

In this section, we analyze the key requirements of the real-time big data diagnosis system, extracting the essential features from the literature. Next, we present the key idea of the framework design.

\subsection{Fundamental prerequisite for diagnosing big data processing systems}

In order to design a generic framework for diagnosing big data processing systems, we classified the fundamental requirements of building a diagnosis system on such systems as follows:

- Infrastructure monitoring: Collecting the information about the underlying system, such as network conditions, CPU utilization, memory utilization, and disk I/O status.

- Task execution monitoring: Collecting the task information, including execution time, progress, location, location of its input data, input data size, output data size, CPU/memory usage, and process state (running, waiting, succeeded, failed, killed).

- Abnormal behavior or fault detection: Detecting abnormal behaviors in big data processing systems, such as slowing tasks, failed tasks, very high/low resource usage, and experiencing very high response time for the requests.

- Root-cause analysis: Finding the root cause of performance reduction in big data processing systems, such as the reasons why: tasks are slowing down, resource utilization is low, the response time is high, or when the network latency is high.

- Visualization: Visualizing the collected metrics and the results of root-cause analysis of any failures causing performance reduction in the cluster with a userfriendly interface in real-time.

\subsection{Key design idea}

Motivated by the above-mentioned requirements and inspired by medical diagnosis, we highlight the design idea of root-cause analysis for big data processing systems as shown Fig. 2. which aims to provide holistic monitoring and root cause analysis for big data processing systems. First, a set of Symptom Detectors is defined and developed in Symptom Detection to detect the abnormalities of the big system by processing collected system information stream in real-time. Once a symptom (abnormality) is detected, the Diagnosis Management may launch the corresponding Diagnosers to troubleshoot the cause of the symptom. One symptom may correspond to root causes. Finally, the decisions are made based on the root-cause analysis results.

\subsection{The generalizability of AutoDiagn}

Modern big data processing systems consists of two main types: Big data analytics (e.g., Hadoop, Spark) and Stream processing (e.g., Flink, Spark Stream). Based on our design idea, our AutoDiagn is an independent framework that can be deployed alongside existing big data cluster management systems (e.g., Apache YARN), and ideally it 
is suitable for root-cause analysis of any big data processing system. However, for the scope of this paper and practical certainty, the implementation of AutoDiagn focuses on debugging root causes of performance degradation (e.g., slow task execution time) in Hadoop due to faults such as data locality, cluster hardware heterogeneity, and network problems (e.g., disconnection). Although we have validated the functionality of AutoDiagn in the context of Hadoop and considering different classes of workload (e.g., WordCount, Grep, TPC-H, TPC-DC, K-means clustering, PageRank), it is generalizable to other big data processing systems executing similar classes of workload.

\section{Autodiagn Architecture}

Following the design idea laid out in \$2, we introduce AutoDiagn, a novel big data diagnosing system. We first illustrate the high-level system architecture and then describe the details of each component. AutoDiagn is implemented in Java and all source code is open-source on GitHub ${ }^{3}$.

\subsection{Architecture overview}

AutoDiagn provides a systematic solution that automatically monitors the performance of big data systems while troubleshooting the issues that cause performance reduction. Fig. 3 shows its two main components: AutoDiagn Monitoring and AutoDiagn Diagnosing. AutoDiagn Monitoring collects the defined metrics (logs) and feeds AutoDiagn Diagnosing with them in real-time. Once the abnormal symptoms are detected by analyzing the collected metrics, a deeper analysis is conducted to troubleshoot the cause of abnormal symptoms.

AutoDiagn Monitoring. AutoDiagn Monitoring is a decentralized real-time stream processing system that collects comprehensive system information from the big data system (e.g., Hadoop Cluster). The Collected Metrics is a set of pre-defined monitoring entities (e.g., CPU usage, memory usage, task location, task status) used to detect the abnormal symptoms. Moreover, the system information, required for understanding the cause of detected abnormal symptoms, is collected in this modular.

AutoDiagn Diagnosing. AutoDiagn Diagnosing is an event based diagnosing system. First, the carefully crafted metrics are injected into the Symptom Detection Engine which is a real-time stream processing module to detect the abnormal symptoms in a big data system. In this paper, we use the outlier which is a common symptom for performance reduction in a Hadoop cluster as a case study to demonstrate the proposed framework. \$4.1 illustrates the details of technology that we developed for symptom detection. Moreover, our system follows the principle of modular programming; the new symptom detection method can be easily plugged in. Diagnoser Plugins is a component for trouble-shooting the reasons behind the detected symptom. A set of Diagnosers is instantiated by the Diagnoser Manager when their corresponding symptoms are detected. Then the instantiated Diagnosers query a time series database to obtain the required input and their outputs illustrate the cause of the detected symptoms.

3. https://github.com/umitdemirbaga/AutoDiagn

\subsection{AutoDiagn monitoring framework}

AutoDiagn monitoring framework is a holistic solution for continuous information collection in a big data cluster. The framework needs to have a fast, flexible and dynamic pipeline to transfer the collected data as well as a high performance, large scale storage system. We now describe an implementation of the framework for a big data computer cluster, and the high-level system architecture is shown in Fig. 4

Information Collection. In each compute node, we develop and deploy an Agent to collect real-time system information. For the worker node, the Agent collects the usage of computing resource via SIGAR APIs ${ }^{4}$ including CPU, memory, network bandwidth, and disk read/write speeds. Moreover, the Agent in the master node collects the usage of computing resource as well as the job and tasks information. The Filter is developed by using GSon Library ${ }^{5}$ to remove the less important information obtained from ResourceManager REST $\mathrm{API}^{\prime}{ }^{6}$ thereby reducing the size of data transmission. The collected information is sent to RabbitM 7 cluster which is a lightweight and easy-to-deploy messaging system in each time interval via Publisher.

Storage. The acquired information is time series data, we therefore choose InfluxDB ${ }^{8}$ for data storage. InfluxDB is a high performance, scalable and open source time series data base which provides a set of flexible open APIs for real-time analytics. The Consumer subscribes the related stream topics from RabbitMQ and interacts with InfluxDB APIs to inject the information to the data base.

Interacting with AutoDiagn Diagnosing. The information required for symptom detection is directly forwarded and processed in AutoDiagn diagnosing via a consumer. If a symptom is detected, InfluxDB will be queried by AutoDiagn diagnosing for root-cause analysis. Finally, the analysis results are sent back to the database to be stored.

User visualization. The user visualization allows the users to have a visible way to monitor their big data system. We utilize InfluxDB's client libraries and develop a set of RESTful APIs to allow the users to query various information, including resource utilization, job and task status, as well as root cause of performance reduction.

\subsection{AutoDiagn diagnosing framework}

In this section, we discuss the core components of the AutoDiagn Diagnosing framework (see Fig. 3), as well as the interactions with each other and the AutoDiagn Monitoring framework.

Symptom Detection Engine. The symptom detection engine subscribes a set of metrics from the real-time streaming system. \$4.1 illustrates the technique that we developed for outlier detection. This component follows microservices architecture to which new symptom detection techniques can be directly attached to our AutoDiagn, interacting with other existing techniques to detect new symptoms.

4. https://github.com/hyperic/sigar

5. https://github.com/google/gson

6. https://hadoop.apache.org/docs/r3.2.1/hadoop-yarn

7. https://www.rabbitmq.com/

8. https://www.influxdata.com/ 


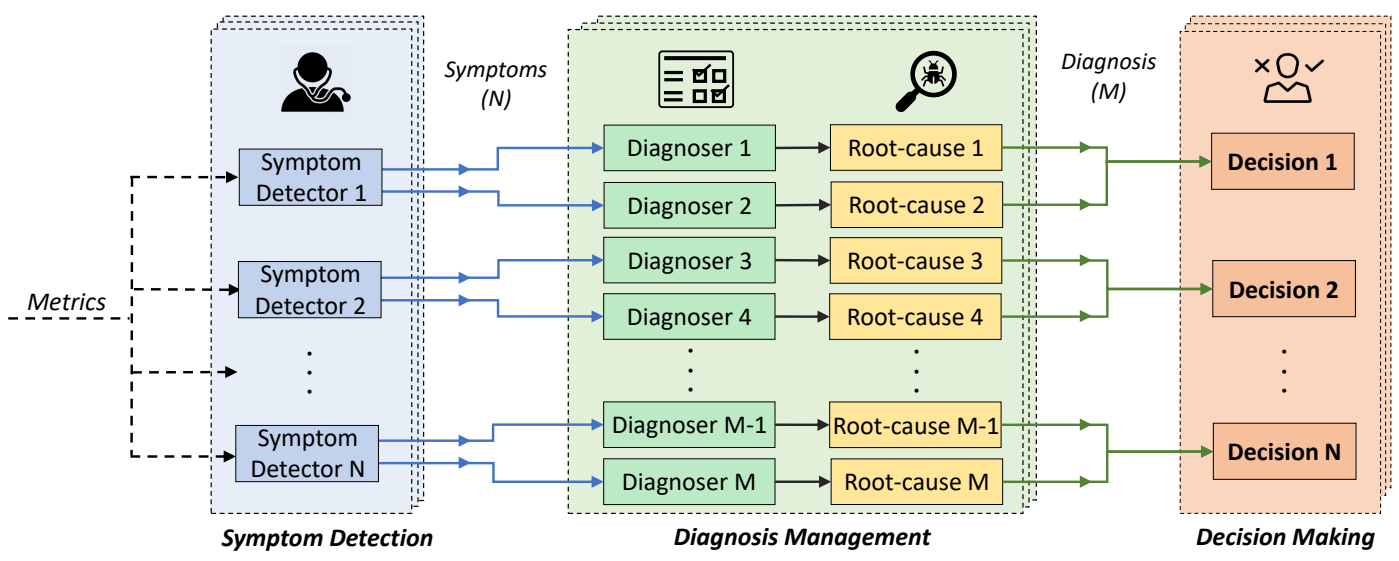

Fig. 2. The key design idea of root-cause analysis for big data processing systems

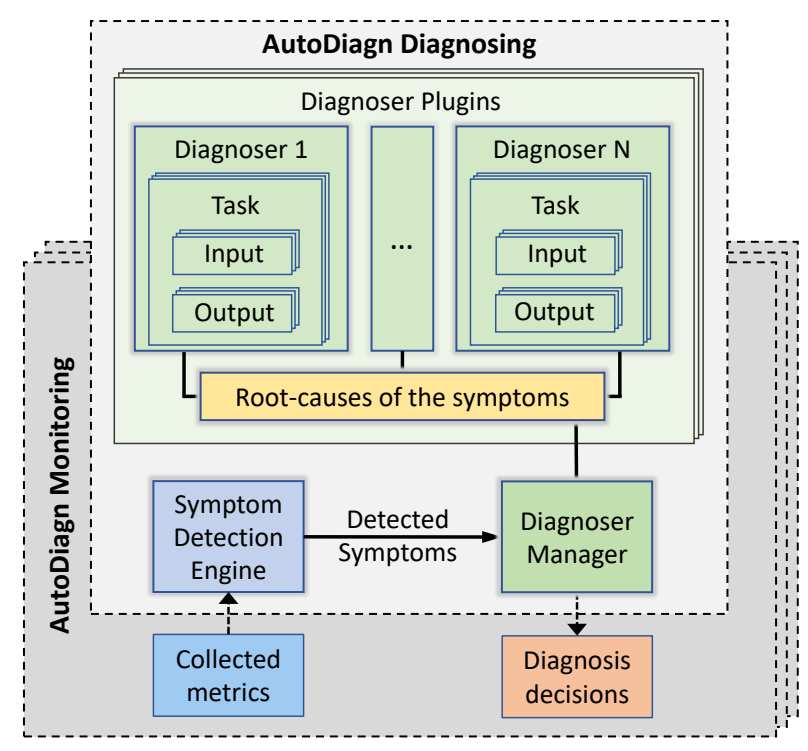

Fig. 3. The high-level architecture of the AutoDiagn system

Diagnoser Manager. The diagnoser manager is the core entity responsible for selecting the right diagnosers to find the reasons that cause the detected symptoms. Additionally, the diagnoser manager is developed as a front-end component, triggered by various detected symptoms (events) via a RESTful API, exposing all diagnosing actions within our framework. The API includes general actions such as starting, stopping or loading a diagnoser dynamically, and specific actions such as retrieving some metrics. Importantly, the diagnoser manager is able to compose a set of diagnosers to complete the diagnosing jobs that may require the cooperation of different diagnosers.

Diagnoser Plugins. The diagnoser plugin contains a set of diagnosers; and a diagnoser is the implementation of the specific logic to perform root-cause analysis of a symptom. Each diagnoser refers to a set of metrics stored in a time series database as the input of its analysis logic. Whenever it is activated by the diagnoser manager, it will perform an analysis, querying the respective metrics, executing the analytic algorithm, and storing the results. $\$ 4.2$ discusses the algorithms to detect the outlier problems, for example, in a
Hadoop cluster. The diagnoser plugin is also designed as a microservice architecture which has two advantages: i) a new diagnoser can be conveniently plugged or unplugged on-the-fly without affecting other components; ii) new rootcause analysis tasks can be composed by a set of diagnosers via RESTful APIs.

\subsection{AutoDiagn diagnosing interfaces for Hadoop}

AutoDiagn exposes a set of simple interfaces for system monitoring, symptom detection and root-cause analysis. Table 1 shows that two types of APIs are defined: highlevel APIs and low-level APIs. The high-level APIs consist of Symptom Detection, Diagnoser and Decision Making. The Symptom Detection APIs are a set of real-time stream processing functions used to detect the defined symptoms causing the performance reduction in the Hadoop system. Each Diagnoser is a query or a set of queries, which aim to find one of the causes of a symptom. For example, QueryNonLocal () tries to find all non-local tasks within a time interval, which is one of the reasons that causes an outlier. Finally, the Decision Making APIs are used to analyze the results from each Diagnoser and make the conclusion. These high-level APIs have to interact with the low-level APIs (Information Collection) to obtain system information including resource usage, and the execution information of the big data system (e.g., ask and job status in a Hadoop system). Based on this flexible design, users can define and develop their own Symptom Detection, Diagnoser and Decision Making APIs and plug them into AutoDiagn.

\subsection{Example applications}

We now discuss several examples for big data system root cause applications using AutoDiagn API.

Outliers. Outliers are the tasks that take longer to finish than other similar tasks, which may prevent the subsequent tasks from making progress. To detect these tasks, the real-time stream query Queryoutlier() is enabled in the Symptom Detection Engine. This function consumes each task's completion rate (i.e., progress) and the executed time to identify the outlier tasks (detailed in \$4.1). Next, three APIs QueryNonlocal(), QueryLessResource() 


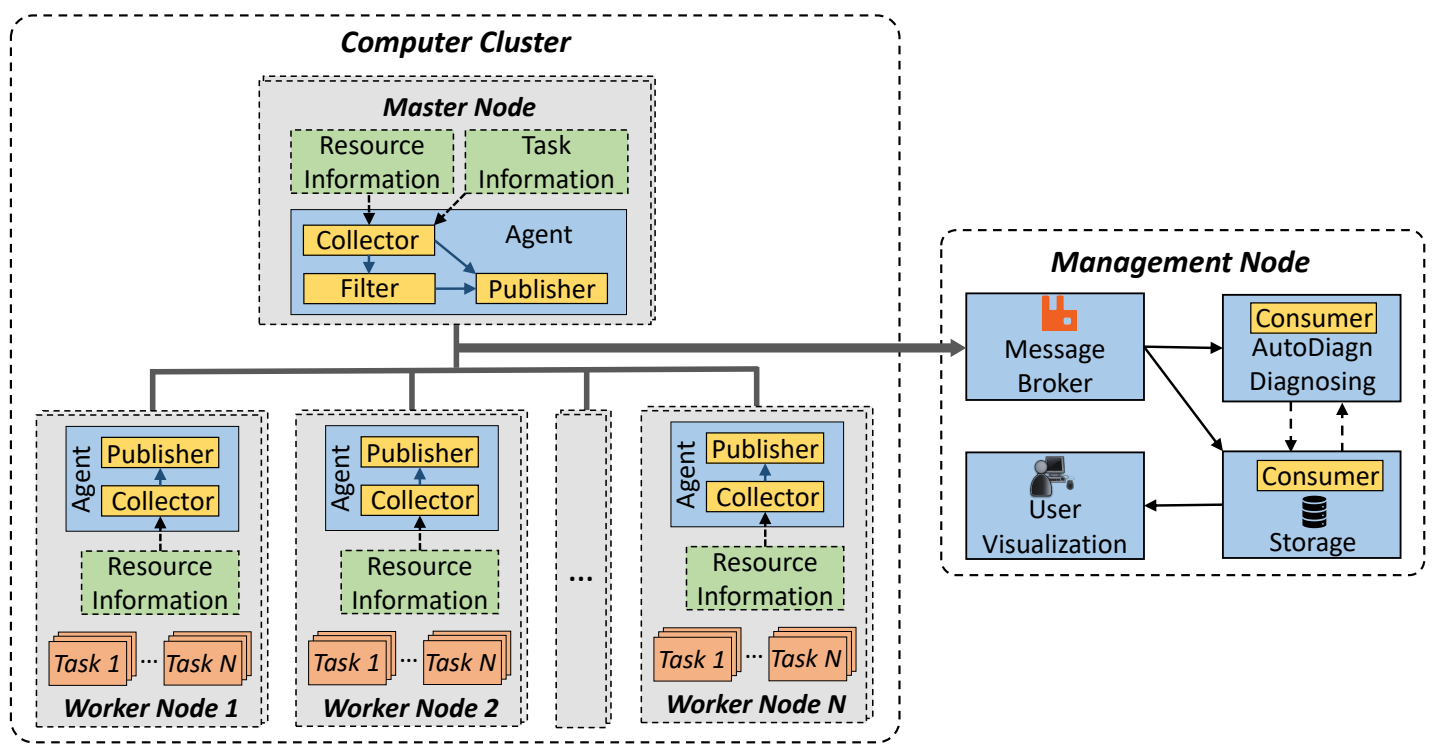

Fig. 4. The high-level architecture of the monitoring framework

and QueryNodeHealth (), corresponding to three Diagnosers that are used to analyze the reasons causing the detected symptom, are executed. QueryNonlocal () queries whether the input data is allocated on the node on which an outlier task is processed. In addition, QueryLessResource () investigates whether outlier tasks are running on the nodes that have less available resource. Moreover, QueryNodeHealth() examines if an outlier task is the task that is a restarted task due to the disconnected nodes from the network. Finally, Rootcauseoutlier() is used to process the results from the three Diagnosers and make the conclusion. All the APIs are shown in Table 1 and the technical details are illustrated in $\$ 4$

Inefficient resource utilization. In our case this means that some tasks are pending (or waiting) to be on worker nodes; at the same time, some worker nodes are idle, e.g., low CPU and memory usage. There are many reasons that cause this issue, but here we consider two key causes: task heterogeneity and resource heterogeneity. The type of tasks in a big data system are various, including CPU intensive tasks, IO intensive tasks and memory intensive tasks. However, the underlying computing resources are typically equally distributed to these tasks, thereby causing inefficient resource utilization. The latter is caused by the heterogeneous underlying computing resources due to the multiple concurrent processing task environments and the queues are built on the saturated nodes.

To detect the inefficient resource utilization in a big data system, the real-time stream query QueryResourceUtil() is used within a defined time interval. We compute the mean and standard deviation of the usage resources of the whole cluster. If the standard deviation is far from the mean, we will further query whether the tasks are queued on the nodes which have high resource usage rates. If inefficient resource utilization is detected, two Diagnosers, QueryOversubscribed() and QueryDiskIOboundTasks(), which are the rootcause analysis APIs shown in Table 1. are executed to perform root-cause analysis. QueryOversubscribed() checks the type of tasks queuing on the saturated nodes. The QueryDiskIOboundTasks () checks whether the saturated nodes have less available computing resource, while processing the allocated tasks. The conclusion of the cause of inefficient resource utilization is made in RootcauseResInef ().

\subsection{Parallel execution}

Following the key design idea, the diagnosers are triggered by the corresponding detected symptom. However, we are able to parallelize the execution of each symptom detector and its diagnosers by partitioning the input data. For example, if one symptom detector needs to process too many data streams, we can use two of the same instances of the symptom detector to process the data streams and aggregate the results from two symptom detectors. The diagnoser can follow the same strategy for parallel execution.

\subsection{Reliability analysis}

AutoDiagn follows the centralized design for data collection, which simplifies the implementation of the Symptom Detection, Diagnosis Management and Decision Making. They can easily obtain the required information from one place, instead of interacting with the entire big data system. Moreover, the centralized design does not mean unreliability, due to the high-availability of RabbitMQ. The RabbitMQ cluster can overcome the node fail in the message queuing system while ensuring scalability.

\section{Case Study}

In the previous section, we have discussed that our framework supports detection of multiple types of symptoms (e.g., outliers, inefficient resource utilization). However, detecting these symptoms is non-trivial; and each symptom can be detected by using different algorithms with different input metrics. In this section, we present a case study that 
TABLE 1

AutoDiagn diagnosing interface. See $\$ 3.4$ for definitions and examples

\begin{tabular}{|c|c|}
\hline Symptom Detection (High-level APIs) & Description \\
\hline QueryOutlier() & Execute a Query that returns the list of out liers if any. \\
\hline QueryResourceutil() & $\begin{array}{l}\text { Execute a Query that returns the list of the worker nodes in which the computing resources are not uti- } \\
\text { lized effectively if any. }\end{array}$ \\
\hline Diagnoser (High-level APIs) & Description \\
\hline QueryNonLocal () & Execute a Query that return the list of non-local tasks if any. \\
\hline QueryLessResource() & Execute a Query that returns false if the cluster is not homogeneous in terms of having resource capacity (CPU/memory). \\
\hline QueryNodeHealth() & Execute a Query that returns the list of disconnected worker nodes in the cluster if any. \\
\hline QueryOversubscribed () & Execute a Query that returns the list of the oversubscribed tasks if any. \\
\hline QueryDiskIOboundTasks() & Execute a Query that returns the list of the disk- or IO-bound tasks if any. \\
\hline Decision Making (High-level APIs) & Description \\
\hline RootcauseOutlier() & Execute a Query that illustrate the main reason of the cause of the outlier. \\
\hline RootcauseResInef () & Execute a Query that illustrate the main reason of the cause of inefficient resource utilization. \\
\hline Information Collection (Low-level APIs) & Description \\
\hline taskExecTime () & Return the execution time since the task started in sec. \\
\hline taskProgress() & Return the progress of the running task as a percentage. \\
\hline taskInput () & Return the input data size of the running task in mb. \\
\hline taskBlock() & Return the block id this task process. \\
\hline taskHost () & Return the name of the node this task ran on. \\
\hline taskCPUusage () & Return the CPU usage of the task. \\
\hline taskMemoryUsage () & Return the memory usage of the task. \\
\hline taskContainerCPU() & Return the allocated $\mathrm{CPU}$ to the container this task ran on. \\
\hline taskContainerMemory () & Return the allocated memory to the container this task ran on. \\
\hline blockHost () & Return the names of the nodes that host the block. \\
\hline pendingTasks() & Return the number of the tasks waiting to be run. \\
\hline nodeTotalCoreNum() & Return the number of the $\mathrm{CPU}$ core number of the node. \\
\hline nodecPuUsage () & Return the $\mathrm{CPU}$ utilization of the node. \\
\hline nodeTotalMem() & Return the total memory capacity of the node. \\
\hline restartedTasks() & Return the name of the restarted tasks due to nodes that got disconnected from the network. \\
\hline nodeMemUsage () & Return the memory utilization of the node. \\
\hline nodeDiskReadSpeed () & Return the disk read speed of the node. \\
\hline nodeDiskWritespeed () & Return the disk write speed of the node. \\
\hline nodeUploadSpeed () & Return the network upload speed of the node. \\
\hline nodeDownloadSpeed () & Return the network download speed of the node. \\
\hline
\end{tabular}

details the technology of detecting outliers and the rootcauses analysis for the detected outliers. The notations used in this paper are summarized in Table 2

TABLE 2

A summary of symbols used in the paper

\begin{tabular}{|l|l|}
\hline Symbols & Description \\
\hline$J_{p}$ & Job progress \\
\hline $\mathcal{N}$ & Name of the task \\
\hline$N_{l}$ & List of $\mathcal{N}$ \\
\hline $\mathcal{P}$ & Performance of the $\mathcal{N}$ \\
\hline$P_{l}$ & List of $\mathcal{P}$ \\
\hline $\mathcal{O}$ & Progress of the $\mathcal{N}$ \\
\hline$O_{l}$ & List of $\mathcal{O}$ \\
\hline $\mathcal{T}$ & Execution time of the $\mathcal{N}$ \\
\hline$T_{l}$ & List of $\mathcal{T}$ \\
\hline$m_{e d}$ & The performance of median task \\
\hline $\mathcal{D}$ & Non-local tasks \\
\hline$D_{l}$ & List of Non-local task \\
\hline $\mathcal{R}$ & Task running on the node with less resources \\
\hline$R_{l}$ & List of $\mathcal{R}$ \\
\hline $\mathcal{W}$ & Restarted tasks due to the nodes' network failure \\
\hline$W_{l}$ & List of $\mathcal{W}$ \\
\hline$S_{l}$ & List of outlier task \\
\hline$S d$ & Non-local outlier \\
\hline$S d_{l}$ & List of $S d$ \\
\hline$S r$ & Outlier stemming from the resource variation \\
\hline$S r_{l}$ & List of $S r$ \\
\hline$S w$ & Outlier stemming from disconnected nodes \\
\hline$S w_{l}$ & List of $S w$ \\
\hline $\mathcal{F}$ & Factor value of 1.5 used to find the $\mathcal{S}$ \\
\hline
\end{tabular}

\subsection{Symptom detection for outliers}

Ananthanarayanan et al. [10] defined the outlier tasks' runtime to be 1.5 times higher than that of the median task execution time; their method is based on the assumption that all tasks are started at the same time and are the same type (i.e., the same input data and the same processing code), which is not suitable for real-time symptom detection, because in a time interval the tasks may be submitted at different times; the input data size of the tasks and the code for tasks are not always the same. In this paper, we use Performance $(\mathcal{P})$ to measure the outlier as shown in Eq $1 . \mathcal{O}$ represents the normalized value of the task progress in terms of percent work complete, and $\mathcal{T}$ is the normalized value of the task execution time.

$$
\mathcal{P}=\frac{\mathcal{O}}{\mathcal{T}}
$$

Eq 2 is used to normalize the $\mathcal{O}$ and $\mathcal{T}$, where $x_{\text {min }}$ and $x_{\max }$ are the minimal and maximal values of the given metrics (eg., task progress and execution time) in a time interval. We set $b=1$ and $a=0.1$ to restrict the normalized values within the range from 0.1 to 1 [12].

$$
x_{n o r m}=a+\frac{\left(x-x_{\min }\right)(b-a)}{x_{\max }-x_{\min }}
$$

Moreover, we define the outlier tasks which have 1.5 times less performance value than the median performance value in each time interval. Fig. 5 shows a snapshot of a time 


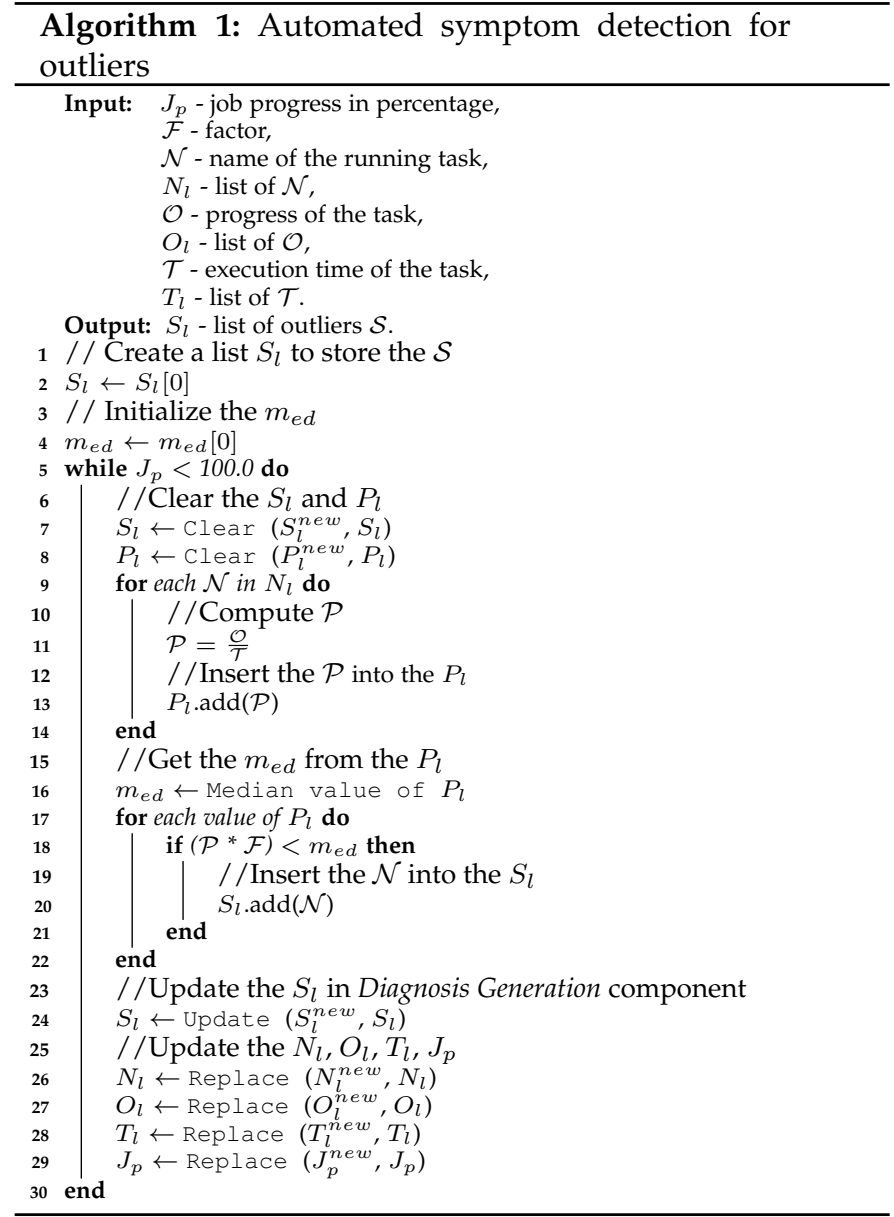

interval (e.g., three seconds), and two mappers are identified as outliers. More evaluations will be discussed in $\$ 5$

Algorithm 1 demonstrates the proposed $A S D$ (automated symptom detection) algorithm in the AutoDiagn system. It is fed by the streaming data provided by the AutoDiagn Monitoring system during job execution. First, the performance of each running task is calculated (see Algorithm 1. Line 11) using Eq 1 Next, the median value of the performance of all tasks is taken to be used to detect outliers (see Algorithm 17. Line 16). Then, the tasks whose performance is 1.5 times less than the performance of the median task are selected as outliers (see Algorithm 11. Line 20. . As a final step, these tasks detected as outliers are sent to the Diagnosis Generation component for root-cause analysis (see Algorithm 1. Line 24).

\subsection{Root cause analysis for outliers}

When the detected symptoms are passed to the Diagnoser Manager, the corresponding Diagnosers are executed for trouble-shooting. The following subsection illustrates the technologies that we have developed for analyzing the causes of outliers in a Hadoop cluster.

\subsubsection{Root cause of outliers}

In this paper, we follow the three main reasons that cause outliers, discussed in [10], i.e., Data locality, Resource heterogeneity, and Network failures.

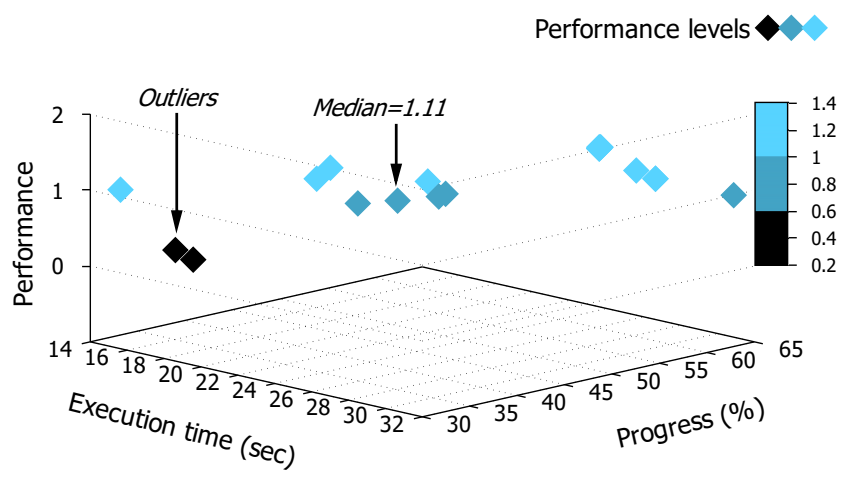

Fig. 5. Performance evaluation of the tasks

Data locality. Hadoop Distributed File System (HDFS) stores the data in a set of machines. If a task is scheduled to a machine which does not store its input data, moving data over the network may introduce some overheads to cause the outliers issue.

Resource heterogeneity. The machines in a Hadoop cluster may be homogeneous with the same hardware configuration, but the run-time computing resources are very heterogeneous due to the multiple talents environment, multiple concurrent processing task environment, machine failures, machine overloaded etc. If a task is scheduled to a bad machine (e.g., has less computing resource) it may cause an outlier issue. Moreover, resource management systems for a large-scale cluster like YARN split the tasks over the nodes equally without considering the resource capacities of the nodes in the cluster, but only takes into account sharing the node's resources among the tasks running on the node equally by default [13]. That is more likely to raise an outlier problem in the cluster.

Network failure. In Hadoop clusters, the network disconnection can cause the running tasks allocated on a disconnected node to be restarted on other nodes, which may lead to the task becoming an outlier and, increase the completion time. The following illustrates the three algorithms that we developed to identify the outliers caused by the three reasons.

\subsubsection{Detecting data locality issues}

We assume that a non-local task $(\mathcal{D})$ (e.g., mapper) is executed on a node where its input data is not stored (In the following, we use $S \boldsymbol{d}$ to represent non-local outliers). To detect these tasks, we develop Algorithm 2 to check whether a set of outliers is caused by a data locality issue. The input of our algorithm is a list of detected outliers during the time interval from $t$ to $t+1$ and one of its outputs is a list of outliers which also belongs to the non-local tasks. First, we query our time series database to obtain all non-local tasks within the given time interval (see Algorithm 2, Line 2).

Here, QueryNonLocal (), a root-cause analysis API, is used to find the non-local ones among the running tasks in that period of time. It compares the location where the task is running (host node of the task) with the nodes where the data block is replicated for fault tolerance via 
information collection APIs shown in Table 1, taskHost () and blockHost (). If the task is not running on any of these nodes (nodes hosting a copy of the block), this task is marked as a non-local task. In the second step (Algorithm 2. Line 4), we obtain the common elements of list $D_{l}$ and $S_{l}$. These elements symbolize the non-local outliers stemming from a data locality issue.

\subsubsection{Detecting resource heterogeneity issues}

Algorithm 2 is designed to identify the outliers caused by the resource heterogeneity. The tasks running on the nodes which have less computing resource $(\mathcal{R})$ tend to be outliers [14] (in the following, we use Sr to represent outliers running on the nodes which have less computing resource). In Algorithm 2. the list of detected outliers during the time interval from $t$ to $t+1$ is used as input and one of the outputs of the algorithm is a list of outliers which also belongs to the tasks running on the node with less computing resource. The time series database is queried to obtain all the tasks running on the node with less computing resource within the given time interval (see Algorithm 2, Line 6).

Here, QueryLessResource(), a root-cause analysis API, is used to check the heterogeneity of the nodes that host only the running tasks based on the resource specifications of them in that period of time. It detects the nodes with less resource capacity in terms of CPU core numbers and the total amount of memory among the nodes hosting the running tasks. The resource specifications of the nodes (i.e., CPU core numbers, total amount of memory) are obtained from each node via information collection APIs shown in Table 1 nodeTotalCoreNum() and nodeTotalMem() APIs. As a second step (Algorithm 2, Line 8, we obtain the common elements of list $R_{l}$ and $S_{l}$. These elements symbolize the outliers stemming from a cluster heterogeneity issue.

\subsubsection{Detecting network failure issues}

Since $S_{l}$ is obtained from Algorithm 1, a Diagnoser is executed via QueryNodeHealth ( ) to find all restarted tasks due to the nodes disconnected by network failure within the given time interval (see Algorithm 2. Line 10). The low-level API restartedTasks () is called which distinguishes the restarted tasks due to network failure from the speculation of straggler tasks by analyzing the information of the tasks that is provided by the monitoring agent. Thereafter, we compute the list $S w_{l}$ that contains the outlier tasks caused by the network failure (see Algorithm 2, Line 12).

\subsubsection{Decision making}

In this case study, we use a simple decision make method that compares the lists $S d_{l}, S r_{l}$ and $S w_{l}$ and the probability of the reasons causing the outliers by using the number of the elements of a list divided the total number of outlier tasks. For instance, the probability of the performance reduction caused by data locality is $\frac{\left|S d_{l}\right|}{\left|S_{l}\right|}$. More advanced methods such as deep learning models can be used for processing more complicated decision making tasks in future work.

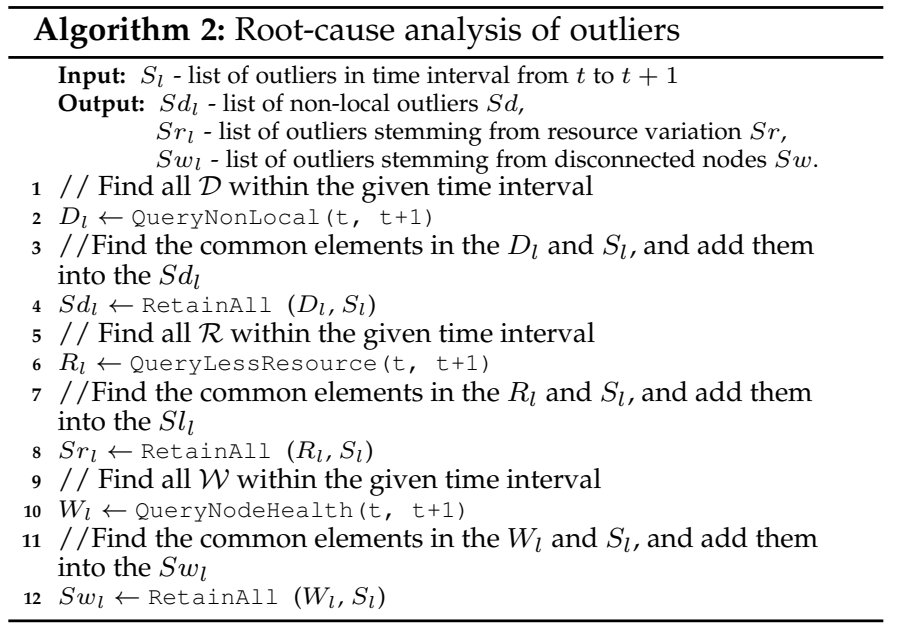

\section{Evaluation}

In this section, we present a comprehensive evaluation showing the capacity and the accuracy rate of AutoDiagn, as well as a analysis of its resource consumption and overheads.

\subsection{Experimental setup}

Environments. We set up the Hadoop YARN clusters over 31 AWS nodes with 1 master and 30 slaves with the Operating system of each node being Ubuntu Server 18.04 LTS (HVM). The Hadoop version is 3.2.1 and the Hive version is 3.1.1. To meet our experimental requirements, we built two types of cluster. In Type I each node has the same configuration (i.e., 4 cores and 16 GB memory). In Type II, 25 nodes have 4 cores and 16 GB memory and 6 nodes have 2 cores and 4 GB memory.

Benchmarks and workload. We used six well-known Hadoop benchmarks in our evaluations namely: WordCount 9 Grep ${ }^{10}$ TPC-H ${ }^{11}$, TPC-DS ${ }^{12}$, K-means clustering ${ }^{13}$ and PageRank ${ }^{14}$. The input of each benchmark application is 30GB.

Methodology. Our experiments aim to evaluate the effectiveness of AutoDiagn. To this end, we manually inject the above-mentioned three main reasons to cause the outliers, which can be summarized as three types of execution environment. Env $\mathcal{A}$ : we perform all benchmark experiments in the cluster Type I. Env $\mathcal{B}$ : we perform all benchmark experiments in the cluster Type I, but skew the input size stored on different nodes. Env $\mathcal{C}$ : we perform all benchmark experiments in the cluster Type II (a heterogeneous cluster). Env $\mathcal{H}$ : we perform all benchmark experiments in the cluster Type I, and disconnect some nodes' network during execution. Each benchmarking is repeated 5 times and results are reported as the average and standard deviation. In total, there are 90 experiments conducted in our evaluation.

\footnotetext{
9. http://wiki.apache.org/hadoop/WordCount

10. http://wiki.apache.org/hadoop/Grep

11. http://www.tpc.org/tpch/

12. http://www.tpc.org/tpcds/

13. https://en.wikipedia.org/wiki/K-means_clustering

14. https://en.wikipedia.org/wiki/PageRank
} 
TABLE 3

The accuracy of symptom detection for non-local outliers in a homogeneous cluster

\begin{tabular}{|c|c|c|c|c|c|}
\hline Benchmark & $\begin{array}{c}\text { Total } \\
\text { tasks }\end{array}$ & $\mathcal{D}$ & $\begin{array}{c}\text { Outliers } \\
\text { (detected as } \mathbf{S d} \text { ) }\end{array}$ & $\begin{array}{c}\text { Accuracy } \\
\mathbf{( \% )}\end{array}$ & $\begin{array}{c}\text { Error } \\
(\boldsymbol{\sigma})\end{array}$ \\
\hline WordCount & 234 & 32 & 29 & 90.63 & 3.9 \\
\hline Grep & 236 & 37 & 33 & 89.19 & 4.8 \\
\hline TPC-H & 102 & 13 & 12 & 92.31 & 6.72 \\
\hline TPC-DS & 126 & 13 & 12 & 92.31 & 6.1 \\
\hline K-means & 234 & 34 & 29 & 85.29 & 1.25 \\
\hline PageRank & 235 & 28 & 25 & 89.29 & 6.2 \\
\hline
\end{tabular}

TABLE 4

The accuracy of symptom detection for the outliers stemming from resource variation in a heterogeneous cluster

\begin{tabular}{|c|c|c|c|c|c|}
\hline Benchmark & $\begin{array}{c}\text { Total } \\
\text { tasks }\end{array}$ & $\mathcal{R}$ & $\begin{array}{c}\text { Outliers } \\
\text { (detected as } \text { Sr) }\end{array}$ & $\begin{array}{c}\text { Accuracy } \\
\mathbf{( \% )}\end{array}$ & $\begin{array}{c}\text { Error } \\
(\sigma)\end{array}$ \\
\hline WordCount & 234 & 37 & 33 & 89.19 & 2.77 \\
\hline Grep & 236 & 26 & 24 & 92.31 & 4.77 \\
\hline TPC-H & 102 & 9 & 8 & 88.89 & 5.47 \\
\hline TPC-DS & 126 & 13 & 12 & 92.31 & 6.9 \\
\hline K-means & 234 & 36 & 33 & 91.67 & 2.88 \\
\hline PageRank & 235 & 30 & 28 & 93.33 & 5.35 \\
\hline
\end{tabular}

\subsection{Diagnosis detection evaluation}

In this section, we evaluate the accuracy of our symptom detection method. To this end, we execute our benchmarks in Env $\mathcal{B}$ to increase number of $\boldsymbol{S} \boldsymbol{d}$ tasks (see $\$ 4.2 .2$ ). Next, to increase the issue of resource heterogeneity ( $\boldsymbol{S r}$ referring to 4.2 .3 , we run the benchmarks in Env $\mathcal{C}$. Thereafter, we run the benchmarks in Env $\mathcal{H}$ to emulate the network failure (Sw referring to $\$ 4.2 .4$. Finally, we compare the detected Outlier tasks with the ground truths that are the data locality, resource heterogeneity, and network failure issues observed by the AutoDiagn diagnosing system.

Table 3, Table 4 , and Table 5 summarize all the results. All benchmarks achieve high accuracy by using our proposal symptom detection method. The highest accuracy for both $S \boldsymbol{d}$ and $\boldsymbol{S r}$ are $92.3 \%$, and for $S w$ is $94.7 \%$ and the overall accuracy for outlier detection is $91.3 \%$, where the Error represents the variation of the accuracy depending on the repeated experiments.

We compute the accuracy of our symptom detection method by using the number of detected outlier tasks divided by the actual number of the tasks that can cause the outlier issue. Table 3 , for example, $\mathcal{D}$ is the total number of non-local tasks and Outliers $(S d)$ is the number of detected outlier tasks that belong to non-local task. Therefore, the accuracy is $\frac{S d}{\mathcal{D}}$. Table 4 and Table 5 follow the same approach to compute the accuracy.

Outlier verification. To further verify the $S d, S r$, and $S w$ are the main reasons causing the outliers, we conduct the following comparison experiments: 1) comparing the execution time of local tasks and non-local tasks; 2) comparing the execution time of the tasks running in Env $\mathcal{A}$ and Env $\mathcal{C}$; and 3) comparing the execution time of normal tasks and restarted tasks due to network failure. Fig. 6(a) proves that non-local tasks consume more time than local tasks due to the overload introduced by data shuffling. Additionally, we
TABLE 5

The accuracy of symptom detection for the outliers stemming from network failures

\begin{tabular}{|c|c|c|c|c|c|}
\hline Benchmark & $\begin{array}{c}\text { Total } \\
\text { tasks }\end{array}$ & $\mathcal{W}$ & $\begin{array}{c}\text { Outliers } \\
\text { (detected as } \mathbf{S w} \text { ) }\end{array}$ & $\begin{array}{c}\text { Accuracy } \\
\mathbf{( \% )}\end{array}$ & $\begin{array}{c}\text { Error } \\
(\sigma)\end{array}$ \\
\hline WordCount & 234 & 11 & 10 & 90.91 & 1.83 \\
\hline Grep & 236 & 13 & 12 & 92.31 & 6.73 \\
\hline TPC-H & 102 & 13 & 12 & 92.31 & 6.54 \\
\hline TPC-DS & 126 & 15 & 14 & 93.33 & 5.43 \\
\hline K-means & 234 & 17 & 16 & 94.12 & 4.33 \\
\hline PageRank & 235 & 19 & 18 & 94.74 & 4.23 \\
\hline
\end{tabular}

compare the throughput of the local tasks and non-local tasks in terms of how much data can be processed in each second. Fig. 7 7 reveals that the throughput of non-local tasks is only $70 \%$ that of local tasks.

Moreover, Fig. 6(b) shows that the execution time of the tasks running on Env $\mathcal{A}$ is less than that on Env $\mathcal{C}$. This is because the tasks are equally distributed to all computing nodes and the less powerful nodes are saturated. Furthermore, Fig. 9(a) shows that the CPU usage of less powerful hosts reaches $100 \%$, thereby building a task queue in these hosts, increasing the overall execution time. However, Fig. 9(b) reveals that the powerful hosts have sufficient computing resources for processing the allocated tasks.

Furthermore, Fig. 6(c) shows that the execution time of the restarted tasks are longer than the normal tasks. As Fig. 8 illustrates, we compute the execution time of the restarted task by adding the execution time of the task in the disconnected node and that in the rescheduled node.

\subsection{Performance and overheads}

Performance evaluation. We evaluate the performance of AutoDiagn by measuring the end-to-end response time of symptom detection and root-cause analysis. Since they are not affected by the types of benchmark, we report the average of the response time. Fig. 10(a) shows that the real-time symptom detection can achieve a low response time, which only has 96 milliseconds and 1059 milliseconds with 100 tasks and 1000 tasks, respectively. Although the response time increases linearly, the parallel execution method discussed in $\$ 3.6$ can be applied to reduce the latency. The response time for root cause analysis is higher than that of symptom detection. For 100 tasks and 1000 tasks, their response times are 0.354 seconds and 5.974 seconds, respectively. Unlike the symptom detection which is very sensitive to latency because of the follow-up processes, triggering the further root-cause analysis or alerting the system managers, Root-cause analysis aims to provide a holistic diagnosing of a big system and the analysis results may help to improve the system performance in future. As a result, the real-time root-cause analysis is not compulsory.

System overheads. To evaluate the system overhead introduced by AutoDiagn, we measure the CPU and memory usage of AutoDiagn Monitoring (agent) and AutoDiagn Diagnosing. Table 6 shows that -AutoDiagn Monitoring only consumes approximately $2.52 \%$ memory and $4.69 \%$ CPU; while -AutoDiagn Diagnosis uses $2.08 \%$ memory and 3.49\% CPU. 


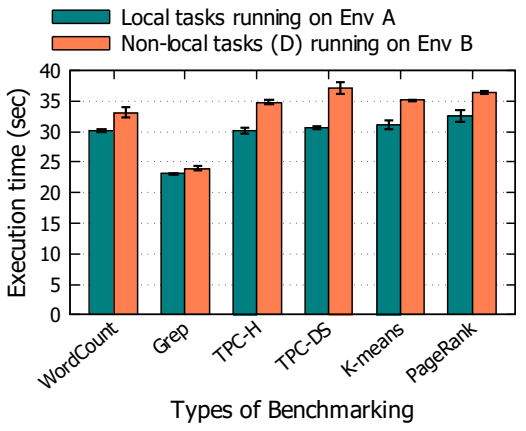

(a) Local tasks vs Non-local tasks

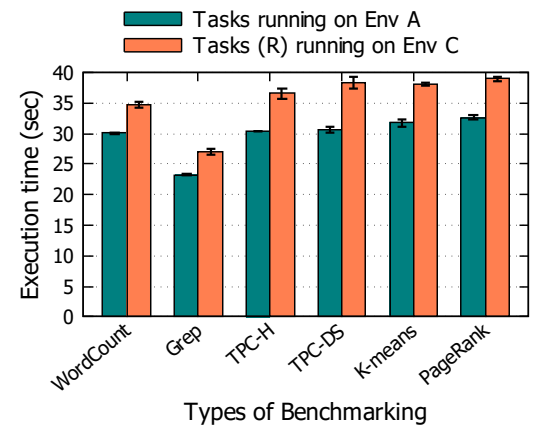

(b) Homogeneous cluster vs Heterogeneous (c) Normal tasks vs Restarted tasks caused by cluster

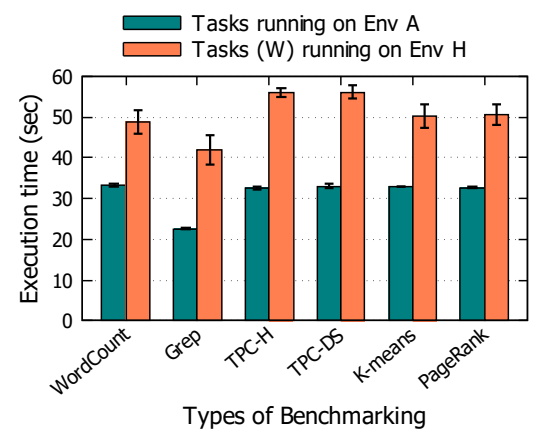

network failure

Fig. 6. Comparison of execution time of the tasks

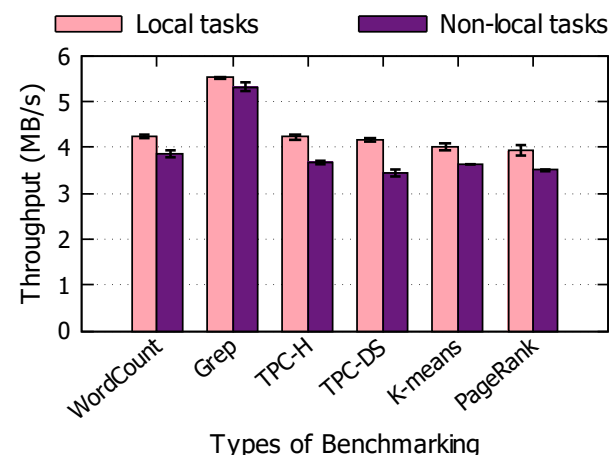

Fig. 7. The throughput of AutoDiagn

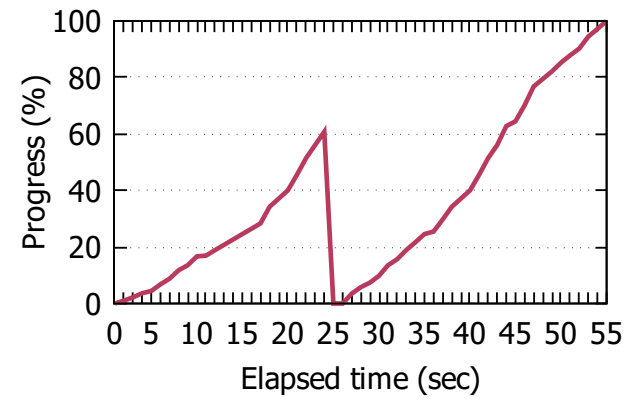

Fig. 8. The life cycle of the restarted task

Fig 10(b) shows the network overhead of AutoDiagn. The extra communication cost introduced by our tool is small but it increases when the number of parallel tasks increases. For example, when the number of parallel task is 100 , there are about 45 messages per second sent from agents to RabbitMQ cluster and the total size of these messages is $13.5 \mathrm{~KB} / \mathrm{s}$. The message rate and network overhead increase to 615 per second and $223 \mathrm{~KB} / \mathrm{s}$, respectively, when the number of parallel tasks is 1000 .

Storage overheads. AutoDiagn needs to dump the system information to a database which may consume extra storage resource. In our evaluation experiments, it only cost 3.75 MB disk space in total. Obviously, increasing the types of symptom detection and root cause analysis will also consume more storage resources. We discuss the potential
TABLE 6

Resource overhead caused by AutoDiagn components

\begin{tabular}{c|c|c} 
Components & Mem (\%) & CPU (\%) \\
\hline AutoDiagn Monitoring & 2.52 & 4.69 \\
AutoDiagn Diagnosing & 2.08 & 3.49
\end{tabular}

future work in $\$ 6$

\section{Discussion And Future Work}

Populating applications. In this paper, we propose a general and flexible framework to uncover the performance reduction issues in a big data system. In particular, we develop and evaluate big data applications for outliers. New applications (including symptom detection and root-cause analysis) are required to populate our system for future work.

Overhead cost reduction. Our system is designed in a loosely-coupled manner, the processing components can be easily scaled. However, the storage overhead increases with the number of applications increasing. [15] proposed a caching method to aggregate the information before sending to destination nodes. We will explore this direction in future work to reduce the storage overhead and network overhead.

Performance improvement. Mantri [10] utilized the outputs of the root cause analysis to improve the resource allocation in Hadoop clusters. Thus, one open research direction is to build a system which can react to analysis results, thereby improving the performance of the big data system.

\section{Related Work}

Much recent work in big data systems focuses on improving workflows [16], [17], [18], programming framework [19], [20], [21], task scheduling [22], [23], [24].

Root-cause analysis. There is a large volume of published studies describing the role of root-cause analysis. The authors of [10], [25], [26] take the next step of understanding the reasons for performance reduction. Mantri [10] characterizes the prevalence of stragglers in Hadoop systems as well as troubleshooting the cause of stragglers. Dean and Barroso [25] analyze the issues causing tail latency in big 


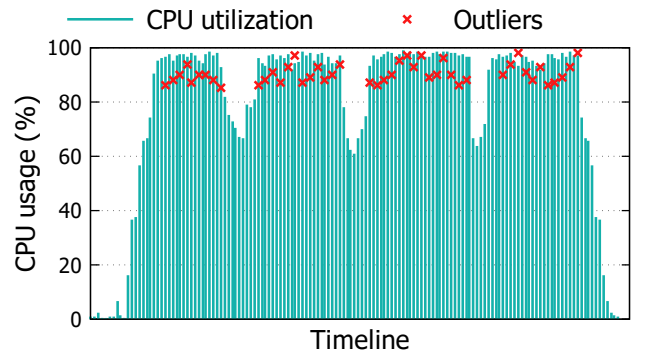

(a) CPU utilization of less powerful hosts and outliers

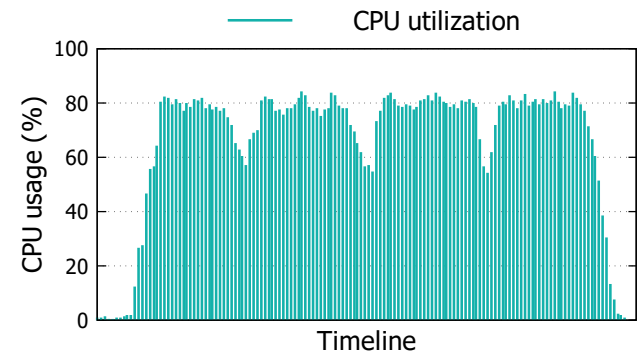

(b) CPU utilization of high power hosts

Fig. 9. CPU utilization of two nodes running simultaneously. Outliers are most likely to occur in the nodes which have less computing resource.

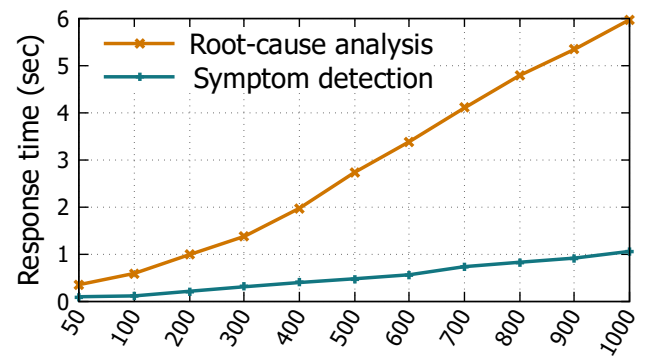

Number of tasks running in parallel

(a) The end-to-end response time of AutoDiagn diagnosis system

Fig. 10. Performance evaluation and network overhead of AutoDiagn

data systems. Garraghan et al. [11], [27] proposed a new method to identify long tail behavior in big data systems and evaluated in google data trace. The authors in [28] use offline $\log$ analysis methods to identify the root cause of outliers in a large-scale cluster consisting of thousands of nodes by tracking the resource utilization. Similarly, Zhou et al. [29] use a simple but efficient rule based method to identify the root cause of stragglers.

Along with these similar works, there are some researchers using statistical and machine learning methods for root-cause analysis. The authors of [30] introduce a Regression Neural Network (RNN) based algorithm to troubleshoot the causes of stragglers by processing Spark logs. More algorithms such as the associated tree and fuzzy data envelopment analysis [31] and Reinforcement Learning [32] are applied for finding the reasons of stragglers in Hadoop and Spark.

In [33], a Pearson coefficient of correlation is used for root cause analysis to measure linear correlation between system metrics, workload and latency. However, these works lack a systematic solution for root cause analysis for big data processing systems and the proposed methods are not applicable for real-time systems.

Different to other work, the authors of [34] propose a new algorithm that aims to reduce the proportion of straggler tasks in machine learning systems that use gradientdescent-like algorithms. This work offers an idea to develop new Diagnosers for machine learning systems using our framework.

Anomaly detection and debugging. The authors in [35] propose a rule-based approach to identify anomalous behaviors

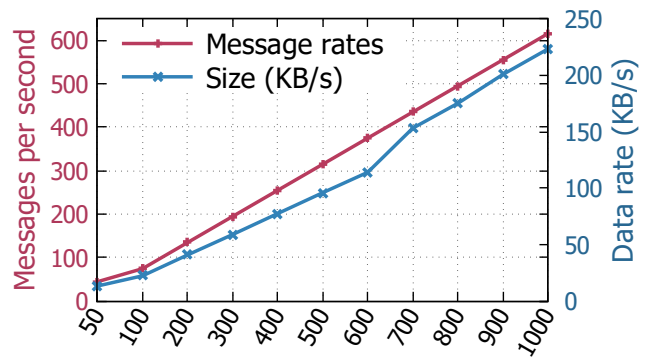

Number of tasks running in parallel

(b) The message rates and network overhead

in Hadoop ecosystems by analyzing the task logs. This work only analyzes the task logs, which fails to capture the performance reduction issues caused by inefficient utilizing the underlying resources. Next, Khoussainova et al. 36] build a historical log analysis system to study and track the MapReduce jobs which cause performance reduction based on their relevance, precision and generality principles. However, this cannot be performed for real-time anomaly detection. Du et al. [37] train a machine learning model from the normal condition data by using Long Short-Term Memory (LSTM) and this trained model is used for detecting in Hadoop and OpenStack environments. Our AutoDiagn provides infrastructure into which the trained models can be plugged to enrich the applications.

Real-time operational data analytic system. Agelastos et al. [38] propose a monitoring system for HPC systems, which can capture the cases of applications competing for shared resources. However, this system does not consider rootcause analysis of the performance reduction. The authors of [5], 39] do not only provide the feature of real-time monitoring, but are also able to identify the performance issues and trouble-shoot the cause of the issues. In addition to them, [40] uses a type of artificial neural network called autoencoder for anomaly detection. They first monitor the system in real-time and collect the normal data for training the model used to discern between normal and abnormal conditions in an online fashion. However, these systems are developed for HPC clusters and are not suitable for big data systems.

Table 7 presents a brief overview of various monitoring tools for big data frameworks. 
TABLE 7

The features supported by existing tools and AutoDiagn

\begin{tabular}{|c|c|c|c|c|c|c|c|c|c|c|c|}
\hline Feature & $\begin{array}{l}\text { DataDog } \\
\text { |2 }\end{array}$ & $\begin{array}{l}\text { Sequence } \\
\text { IQ [3] }\end{array}$ & $\begin{array}{l}\text { Sematext } \\
{[4]}\end{array}$ & $\begin{array}{l}\text { TACC } \\
\mid 5\end{array}$ & $\begin{array}{l}\text { Mantri } \\
\text { 10 }\end{array}$ & $\begin{array}{l}\text { DCDB } \\
\text { |39] }\end{array}$ & $\begin{array}{l}\text { Nagios } \\
{[41]}\end{array}$ & $\begin{array}{l}\text { Ganglia } \\
42\end{array}$ & $\begin{array}{l}\text { Chukwa } \\
\text { 43 }\end{array}$ & $\begin{array}{l}\text { DMon } \\
\text { [44] }\end{array}$ & AutoDiagn \\
\hline $\begin{array}{l}\text { BigData frameworks } \\
\text { support }\end{array}$ & Good & Poor & Good & No & Poor & No & Poor & Poor & Poor & $\begin{array}{l}\text { Good and } \\
\text { Extensible }\end{array}$ & $\begin{array}{l}\text { Good and } \\
\text { Extensible }\end{array}$ \\
\hline $\begin{array}{l}\text { Underlying resource } \\
\text { monitoring }\end{array}$ & Yes & Yes & Yes & Yes & Yes & Yes & Yes & Yes & Yes & Yes & Yes \\
\hline Auto-scaling & Yes & Yes & Yes & Yes & Yes & Yes & No & No & Yes & Yes & Yes \\
\hline Alerts & Yes & No & Yes & No & No & No & Yes & No & No & No & Yes \\
\hline $\begin{array}{l}\text { Visualization of big } \\
\text { data tasks }\end{array}$ & Yes & No & Yes & No & No & No & No & Yes & No & No & Yes \\
\hline $\begin{array}{l}\text { User customized } \\
\text { root-cause analysis }\end{array}$ & No & No & No & No & No & No & No & No & No & No & Yes \\
\hline
\end{tabular}

\section{CONCLUSION}

In this paper, we have presented AutoDiagn, a framework for enabling diagnosing of large-scale distributed systems to ascertain the root cause of outliers, with the core purpose of unravelling the concretization of complicated models for system management. After making a comprehensive literature review and identifying the requirements for realworld problems, we conceived its design. The combination of user-defined functions powered by APIs and the agentbased monitoring system along with the findings obtained from an empirical analysis of the experiments we conducted play a fundamental role in the development of the system. AutoDiagn can be applied to most big data systems along with the monitoring systems. We have also presented the implementation and integration of the AutoDiagn system to the SmartMonit [45], real-time big data monitoring system, combined in our production environment. In our implementation on a large cluster, we find AutoDiagn very effective and efficient.

Outliers are one of the main problems in big data systems that overwhelm the whole system and reduce performance considerably. AutoDiagn embraces this problem to reveal the bottlenecks alongside their root causes.

\section{ACKNOWLEDGEMENT}

This research is funded by the Turkish Ministry of National Education. This research is partially funded by the following UKRI projects: SUPER (EP/T021985/1), PACE (EP/R033293/1), and Centre for Digital Citizens (EP/T022582/1). This work is also supported by the grant of National Natural Science Foundation of China (62072408) and Zhejiang Provincial Natural Science Foundation of China (LY20F020030).

\section{RefERENCES}

[1] A. Noor, K. Mitra, E. Solaiman, A. Souza, D. N. Jha, U. Demirbaga, P. P. Jayaraman, N. Cacho, and R. Ranjan, "Cyber-physical application monitoring across multiple clouds," Computers \& Electrical Engineering, vol. 77, pp. 314-324, 2019.
[2] Datadog. Accessed: 2020-07-13. [Online]. Available: https: //www.datadoghq.com/

[3] Sequenceiq. Accessed: 2020-07-14. [Online]. Available: https: //github.com/sequenceiq

[4] Sematext. Accessed: 2020-07-13. [Online]. Available: https: //sematext.com/

[5] R. T. Evans, J. C. Browne, and W. L. Barth, "Understanding application and system performance through system-wide monitoring," in 2016 IEEE International Parallel and Distributed Processing Symposium Workshops (IPDPSW). IEEE, 2016, pp. 1702-1710.

[6] G. Iuhasz, D. Pop, and I. Dragan, "Architecture of a scalable platform for monitoring multiple big data frameworks," Scalable Computing: Practice and Experience, vol. 17, no. 4, pp. 313-321, 2016.

[7] I. Drăgan, G. Iuhasz, and D. Petcu, "A scalable platform for monitoring data intensive applications," Journal of Grid Computing, vol. 17 , no. 3, pp. 503-528, 2019.

[8] S. Babu, "Towards automatic optimization of mapreduce programs," in Proceedings of the 1st ACM symposium on Cloud computing, 2010, pp. 137-142.

[9] R. S. Xin, J. Rosen, M. Zaharia, M. J. Franklin, S. Shenker, and I. Stoica, "Shark: Sql and rich analytics at scale," in Proceedings of the 2013 ACM SIGMOD International Conference on Management of data, 2013, pp. 13-24.

[10] G. Ananthanarayanan, S. Kandula, A. G. Greenberg, I. Stoica, Y. Lu, B. Saha, and E. Harris, "Reining in the outliers in mapreduce clusters using mantri." in Osdi, vol. 10, no. 1, 2010, p. 24.

[11] P. Garraghan, X. Ouyang, P. Townend, and J. Xu, "Timely long tail identification through agent based monitoring and analytics," in 2015 IEEE 18th International Symposium on Real-Time Distributed Computing. IEEE, 2015, pp. 19-26.

[12] J. Han, J. Pei, and M. Kamber, Data mining: concepts and techniques. Elsevier, 2011.

[13] T. Renner, L. Thamsen, and O. Kao, "Coloc: Distributed data and container colocation for data-intensive applications," in 2016 IEEE International Conference on Big Data (Big Data). IEEE, 2016, pp. 3008-3015.

[14] A. Rasooli and D. G. Down, "Guidelines for selecting hadoop schedulers based on system heterogeneity," Journal of grid computing, vol. 12, no. 3, pp. 499-519, 2014.

[15] A. Rabkin, M. Arye, S. Sen, V. S. Pai, and M. J. Freedman, "Aggregation and degradation in jetstream: Streaming analytics in the wide area," in 11th \{USENIX\} Symposium on Networked Systems Design and Implementation (\{NSDI\} 14), 2014, pp. 275-288.

[16] Z. Wen, T. Lin, R. Yang, S. Ji, R. Ranjan, A. Romanovsky, C. Lin and J. Xu, "Ga-par: Dependable microservice orchestration framework for geo-distributed clouds," IEEE Transactions on Parallel and Distributed Systems, vol. 31, no. 1, pp. 129-143, 2019.

[17] Z. Wen, J. Cała, P. Watson, and A. Romanovsky, "Cost effective, reliable and secure workflow deployment over federated clouds," IEEE Transactions on Services Computing, vol. 10, no. 6, pp. 929-941, 2016. 
[18] Z. Wen, R. Qasha, Z. Li, R. Ranjan, P. Watson, and A. Romanovsky, "Dynamically partitioning workflow over federated clouds for optimising the monetary cost and handling run-time failures," IEEE Transactions on Cloud Computing, 2016.

[19] G. Malewicz, M. H. Austern, A. J. Bik, J. C. Dehnert, I. Horn, N. Leiser, and G. Czajkowski, "Pregel: a system for large-scale graph processing," in Proceedings of the 2010 ACM SIGMOD International Conference on Management of data, 2010, pp. 135-146.

[20] M. Zaharia, M. Chowdhury, M. J. Franklin, S. Shenker, I. Stoica et al., "Spark: Cluster computing with working sets." HotCloud, vol. 10, no. 10-10, p. 95, 2010.

[21] M. Abadi, P. Barham, J. Chen, Z. Chen, A. Davis, J. Dean, M. Devin, S. Ghemawat, G. Irving, M. Isard et al., "Tensorflow: A system for large-scale machine learning," in 12th \{USENIX\} symposium on operating systems design and implementation (\{OSDI\} 16), 2016, pp. 265-283.

[22] M. Isard, V. Prabhakaran, J. Currey, U. Wieder, K. Talwar, and A. Goldberg, "Quincy: fair scheduling for distributed computing clusters," in Proceedings of the ACM SIGOPS 22nd symposium on Operating systems principles, 2009, pp. 261-276.

[23] N. J. Yadwadkar and W. Choi, "Proactive straggler avoidance using machine learning," White paper, University of Berkeley, 2012.

[24] A. Badita, P. Parag, and V. Aggarwal, "Optimal server selection for straggler mitigation," IEEE/ACM Transactions on Networking, vol. 28, no. 2, pp. 709-721, 2020.

[25] J. Dean and L. A. Barroso, "The tail at scale," Communications of the ACM, vol. 56, no. 2, pp. 74-80, 2013.

[26] K. Ousterhout, R. Rasti, S. Ratnasamy, S. Shenker, and B.-G. Chun, "Making sense of performance in data analytics frameworks," in 12th \{USENIX\} Symposium on Networked Systems Design and Implementation (\{NSDI\} 15), 2015, pp. 293-307.

[27] P. Garraghan, X. Ouyang, R. Yang, D. McKee, and J. Xu, "Straggler root-cause and impact analysis for massive-scale virtualized cloud datacenters," IEEE Transactions on Services Computing, vol. 12, no. 1, pp. 91-104, 2016.

[28] X. Ouyang, P. Garraghan, R. Yang, P. Townend, and J. Xu, "Reducing late-timing failure at scale: Straggler root-cause analysis in cloud datacenters," in Fast Abstracts in the 46th Annual IEEE/IFIP International Conference on Dependable Systems and Networks. DSN, 2016.

[29] H. Zhou, Y. Li, H. Yang, J. Jia, and W. Li, "Bigroots: An effective approach for root-cause analysis of stragglers in big data system," IEEE Access, vol. 6, pp. 41 966-41 977, 2018.

[30] S. Lu, X. Wei, B. Rao, B. Tak, L. Wang, and L. Wang, "Ladra: Log-based abnormal task detection and root-cause analysis in big data processing with spark," Future Generation Computer Systems, vol. 95, pp. 392-403, 2019.

[31] Z. He, Y. He, F. Liu, and Y. Zhao, "Big data-oriented product infant failure intelligent root cause identification using associated tree and fuzzy dea," IEEE Access, vol. 7, pp. 34 687-34 698, 2019.

[32] H. Du and S. Zhang, "Hawkeye: Adaptive straggler identification on heterogeneous spark cluster with reinforcement learning," IEEE Access, vol. 8, pp. $57822-57832,2020$.

[33] J. P. Magalhães and L. M. Silva, "Root-cause analysis of performance anomalies in web-based applications," in Proceedings of the 2011 ACM Symposium on Applied Computing, 2011, pp. 209-216.

[34] R. Bitar, M. Wootters, and S. El Rouayheb, "Stochastic gradient coding for straggler mitigation in distributed learning," IEEE Journal on Selected Areas in Information Theory, vol. 1, no. 1, pp. 277-291, 2020.

[35] A. M. Chacko, J. S. Medicherla, and S. M. Kumar, "Anomaly detection in mapreduce using transformation provenance," in Advances in Big Data and Cloud Computing. Springer, 2018, pp. 91-99.

[36] N. Khoussainova, M. Balazinska, and D. Suciu, "Perfxplain: debugging mapreduce job performance," arXiv preprint arXiv:1203.6400, 2012.

[37] M. Du, F. Li, G. Zheng, and V. Srikumar, "Deeplog: Anomaly detection and diagnosis from system logs through deep learning," in Proceedings of the 2017 ACM SIGSAC Conference on Computer and Communications Security, 2017, pp. 1285-1298.

[38] A. Agelastos, B. Allan, J. Brandt, P. Cassella, J. Enos, J. Fullop, A. Gentile, S. Monk, N. Naksinehaboon, J. Ogden et al., "The lightweight distributed metric service: a scalable infrastructure for continuous monitoring of large scale computing systems and applications," in SC'14: Proceedings of the International Conference for High Performance Computing, Networking, Storage and Analysis. IEEE, 2014, pp. 154-165.

[39] A. Netti, M. Müller, C. Guillen, M. Ott, D. Tafani, G. Ozer, and M. Schulz, "Dcdb wintermute: Enabling online and holistic operational data analytics on hpc systems," in Proceedings of the 29th International Symposium on High-Performance Parallel and Distributed Computing, 2020, pp. 101-112.

[40] A. Borghesi, A. Bartolini, M. Lombardi, M. Milano, and L. Benini, "Anomaly detection using autoencoders in high performance computing systems," in Proceedings of the AAAI Conference on Artificial Intelligence, vol. 33, 2019, pp. 9428-9433.

[41] Nagios. Accessed: 2020-07-15. [Online]. Available: https://www. nagios.org/

[42] Ganglia. Accessed: 2020-07-15. [Online]. Available: http://ganglia. info/

[43] Apache chukwa. Accessed: 2020-07-14. [Online]. Available: https://chukwa.apache.org/

[44] Dmon. Accessed: 2020-07-12. [Online]. Available: https://github. com/Open-Monitor/dmon

[45] U. Demirbaga, A. Noor, Z. Wen, P. James, K. Mitra, and R. Ranjan "Smartmonit: Real-time big data monitoring system," in 2019 38th Symposium on Reliable Distributed Systems (SRDS). IEEE, 2019, pp. 357-3572.

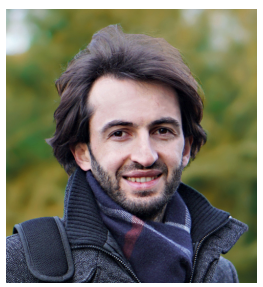

Umit Demirbaga (Member, IEEE) is a PhD student in the School of Computing, Newcastle University, UK. He received an MSc degree in Computer Science from Newcastle University, UK in 2017 and the BSc degree in Electronics and Computer Education from Marmara University, Turkey in 2011. His research interests include big data analytics, cloud computing and distributed systems. He was awarded Outstanding Performance Award with Best Team Project Award in his MSc in 2017.

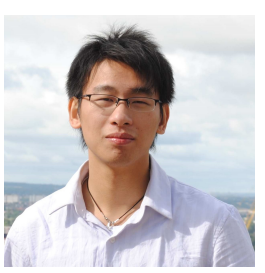

Zhenyu Wen (Member, IEEE) received MSc and $\mathrm{PhD}$ degrees in Computer Science from Newcastle University, Newcastle upon Tyne, UK, in 2011 and 2016, respectively. He is currently a Postdoc Researcher with the School of Computing, Newcastle University, UK. His current research interests include loT, crowd sources, A system, and cloud computing. For his contributions to the area of scalable data management for the Internet of Things. He was awarded the IEEE TCSC Award for Excellence in Scalable Computing (Early Career Researchers) in 2020.

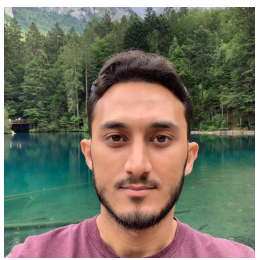

Ayman Noor is a PhD student in Computer Science at Newcastle University, UK. His current research interests include cloud computing monitoring, and machine learning. He earned a Master of Science in Computer and Information Science from Gannon University, PA, USA in 2013 and a Bachelor in Computer Science from the College of Computer Science and Engineering from Taibah University, Madinah, SA in 2006.

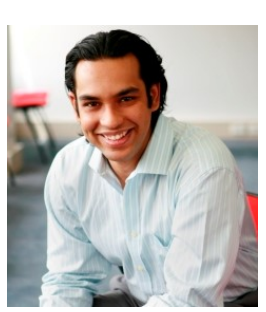

Karan Mitra is an Assistant Professor at Luleå University of Technology, Sweden. He received his Dual-badge PhD from Monash University, Australia and Luleå University of Technology in 2013. His research interests include cloud and mobile cloud computing, performance benchmarking of distributed systems, context-aware computing and QoE. He is a member of the IEEE and ACM. 


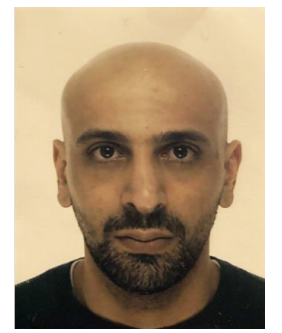

Khaled Alwasel has a BS and MS in information technology from Indiana University-Purdue University Indianapolis (2014) and Florida International University (2015), USA. He is currently working toward a PhD in the School of Computing Science at Newcastle University (UK). Khaled's interests lie in the areas of softwaredefined networking (SDN), big data, loT, edge computing, and cloud computing

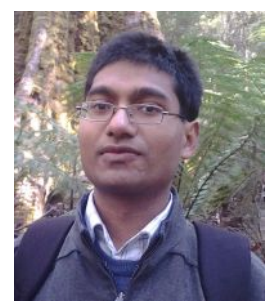

Saurabh Garg is a lecturer at the University of Tasmania, Hobart, Tasmania. He has published more than 30 papers in highly cited journals and conferences with $\mathrm{H}$-index 24 . He has gained about three years of experience in industrial research while working at IBM Research Australia and India. His areas of interest are distributed computing, cloud computing, HPC, IoT, big data analytics, and education analytics.

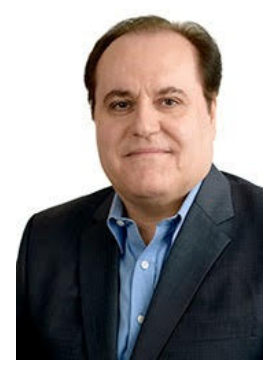

Albert Y. Zomaya is currently the Chair Professor of High Performance Computing \& Networking in the School of Computer Science, University of Sydney. He is also the Director of the Centre for Distributed and High Performance Computing which was established in late 2009. Professor Zomaya was an Australian Research Council Professorial Fellow during 2010-2014 and held the CISCO Systems Chair Professor of Internetworking during the period 2002-2007 and also was Head of School for 2006-2007.

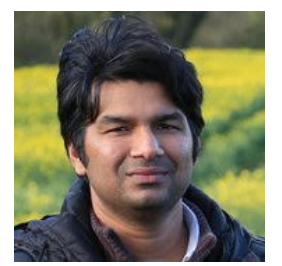

Rajiv Ranjan is a Full professor in Computing Science at Newcastle University, UK. Before moving to Newcastle University, he was Julius Fellow (2013-2015), Senior Research Scientist and Project Leader in the Digital Productivity and Services Flagship of Commonwealth Scientific and Industrial Research Organization (CSIRO C Australian Government's Premier Research Agency). Prior to that he was a Senior Research Associate (Lecturer level B) in the School of Computer Science and Engineering, University of New South Wales (UNSW). Dr Ranjan has a PhD (2009) from the department of Computer Science and Software Engineering, the University of Melbourne. 